\title{
4. Botanisches.
}

\section{Der Kuhbaum.}

Zu den vielen sonderbaren Erscheinungen, welche uns in dem weiten Gebiete der Natur begegnen und uns deren Bestreben zeigen, durch die mannigfachsten Variationen, welche sie in das Walten ihrer Gesetze einfliessen lässt, diesen den Anschein der Monotomie zu benehmen, ohne dabei deren Harmonie Eintrag zu thun, gehören auch jene Baumarten, bei welchen das Niedersteigen der Säfte das Ansehen einer Milchabsonderung anzunehmen scheint. Aus einer dieser milchartigen Flüssigkeiten gewinnt man auch, wenn sie eingetrocknet ist, den Kautschuk. Abgesehen von manchen andern Vegetabilien, deren Säfte äusserlich Aehnlichkeit mit der thierischen Milch zeigen, wie die Euphorbien, der Mohn u. s. w., finden wir eine solche Analogie namentlich bei einem Baume der Cordilleren, der dem Reisenden unter dem Namen Palo de leche, Milchbaum oder Kuhbaum, bekannt ist. Er kommt besonders häufig in der Ungegend von Macaray vor. Macht man in seinen Stamm oder in einen seiner Aeste einen Einschnitt, so quillt ein Saft hervor, der alle innern und äussern Eigenschaften der Milch der 'Thiere zeigt. Als Boussingault sich zu seiner naturwissenschaftlichen Reise nach dem amerikauischen Continent vorbereitete, war es namentlich auch dic Milch des Kubbaums, welche $\mathrm{Hum}$ boldt seiner besonderen Beachtung cmpfahl. Dieser Saft des Kuhbaumes uuterscheidet sich von der Kuhmileh nur dadurch, dass ihm etwas Pflanzenschleim oder Gallerte beigernengt ist, und dass ibn die Säuren gar nicht gerinnen machen, der Alkohol aber nur in geringem Maasse. Unter der Einwirkung gelinder Wärme bildet sich auf seiner Oberfï̈che ein dünnes Häutehen. Lässt man ihn im warmen Wasserbade verdunsten, so erhält man einen dem Frangipan ähnlichen Extract, und bei noch längerer Dauer der Wärme-Einwirkung treten Oeltropfen hervor und nebmen in dem Verbältniss zu, als das Wasser verdunstet; es entsteht dann zuletzt eine fettartige Flüssigkeit, in welcher eine Fasersubstanz, die allmälig unter der Einwirkung der Wärme vertrocknet und horna rtig wird. Gleichzeitig verbreitet sich dann auch ein Duft wie von Fleisch, das in Fett geschmort wird. Es scheidet sich also diese Milch in zwei wesentlich verschiedene Substanzen, in eine flüssige, welche die Eigenschaft des Fettes zeigt, und in einen Faserstoffkörper, der die Figenschaften des Faserstoffes, welcher in Blute der Thiere enthalten ist, besitzt. Der Fettkörper hat viele Aehnlichkeit mit dem Wachs der Bienen. Der Faserkörper schwillt, auf cin erhitztes Eisen gebracht, auf, schmilzt und verwandelt sich, während jener Duft wie von gebratenem Fleische sich entwickelt, in Kohle. Keine der Untersuchungen hatte aber bisher das Vorhandensein des Kautschuks in der Milch des Kuhbaumes nachzuweisen vermocht.

Die Bewolner der Cordilleren trinken, wenn sie sich weit von ihren Wohnungen befinden, häufig die Milch des Kuhbaumes; auch Bousingault und sein Begleiter Rivero tranken sie oft wäbrend ihres Aufentbalts in Macaray. Der genannte Baum ist nach $\mathrm{Hum}$ boldt der Galactodendron dulce, aus der Familie der Verticeen oder des Feigenbaumes. Doch kommen in den Gebirgen, die sich längs des Oceans hinziehen, noch mebrere Baumgattungen vor,

Arch. d. Pharm. CLII. Bds. 2.Hft. 
welche einen milchigen Saft enthalten und die man häufig mit dem Kuhbaume verwechselt. So lïsst auch der in der Limgegend von Macaraibo vorkommende Baum Clusia galactodendron in überreichlicher Menge einen milchähnlichen, sehr angenehm schmeckenden Saft ausfliessen, der indess nicht so viel thierisches Fibrin enthäl, er lässt sich wenigstens nicht so wohl gereinigt herstellen, und findet man ausser jener Wachssubstanz eine viel minder schmelzbare, deren Eigenschaft sie eher den Harzen beizählen lässt. In denselben Landstrichen kommt auch Hura crepitans vor. Der Saft enthält ebenfalls einen stickstoffhaltigen, der thierischen Gallerte ähnlichen Körper. Aber dieser Saft enthält eine krystallisirbare alkalinische Substanz, welche ihn zu einem äusserst intensiven Gifte macht. Man bedient sich seiner in Amerika zum Fischfang, inden man mit ihm das Wasser vergiftet. (Musée des Sciences. - Ausland. 1859. S. 863.) Bkb.

\section{Ein Mauerbrecher aus dem Reiche der Pfanzen.}

Die "India" enthält einige, interessante Angaben über die vernichtende Kraft eines Baumes, dessen Anblick nicht im Entferntesten auf eine so schlimme Begabung schliessen liesse.

Der Papayabaum - sagt sie - kommt sehr häufig in Indien vor, wo er, wie man vermuthet, spontan aufwachsen soll. Er wächst oft an den ungewöhulichsten Stellen empor uod zerstört in seiner weiteren Entwickelung ganze Gebäude durch den heftigen Druck. den seine Wurzeln ausüben, die in dem Kitt, der die Steine und Ziegel auseinander häl, weiter und weiter nach allen Richtungen hin sich ausdehnen: so sieht man ihn oft über Wasserleitungen, über den Kuppeln der Moscheen, über Gartenmauern emporragen. Kein Christ, kein Hindu würde es wagen, die oberen Theile des noch jungen Baumes abzuhauen, und kein Mubamedaner würde sich dazu verstehen. Es würde indess auch eine vergebliche Mübe sein, welche nur das Herannahen jenes verhängnissvollen und unvermeidlichen Tages um Einiges aufschieben könnte. Den $W$ urzeln des Baumes wobnt eine solche Kraft des Wachsthums inne, dass sie unaufhörlich neue $Z$ weige treiben, die, sind sie einmal tief in einen Bau eingedrungen, dann im Innern desselben ihre Angriffe mit einer nicht ermattenden Energie fortsetzen, mit welcher Sorgfalt man auch ibre $\mathbf{Z}$ weige abhauen mag.

Da die Laubkrone des jungen Papayabaumes einen angenehmen Schatten bietet, so beschützen und begünstigen die Indier sogar sein Wachsthum. "Es ist nichts Ueberraschendes, ${ }^{\circ}$ versichert Oberst Sleemann, "dass der Aberglaube diesen anmuthigen, zart geformten Baum den Göttern geweiht habe, denn Puläste, Burgen, Tempel, Grabgewölbe, alle Denkmale, die der Mensch zur Verherrlichung und Verewigung seines Namens aufrichtet und auf die er stolz ist, sie alle zerbröckeln $\mathrm{zu}$ Staub unter jenem furchtbaren Drucke, vor dem nichts sie zu retten mag. Triumphirend richtet er sich uber ihnen empor, und seine prachtvolle Anmuth entfaltend, lässt er unter seinem saftigen grünen Laubwerke die Trümmer der von ihm vollbrachten Zerstörung in Staub zerfallen, als wollte er damit zeigen, wie nichtig die menschlichen Dinge und wie vergeblich all' unsere Anstrengungen seien. Inmittè der Wälder und Gebirge Indiens haust ein göttliches Wesen, dessen Macht die Menschen scheuen und bei dessen Namen sie zu schwören pflegen. Da der Papaya aller Orten den Göttern - von welchen die Sage gebt, 
dass sie es lieben, unter seinem Laubdache zu weilen, nm dessen harmonischen Säuseln zu lauschen - geweibt ist, so nimmt der bei jenem Gott Schwörende eins der Blätter dieses Baumes in die Hand und ruft und fleht zu dem über seinem Haupte befindlichen Gotte, er möge ihn oder die ihn lieb und theuer sind, gleich wie er dieses Blatt in seinen Hünden zerreibe, zermalmen, wenn er jetzt gegen die Wahrheit sprechen werde. Darauf zerreisst, zerbröckelt und zerquetscht der Schwörende das Blatt und bringt dann vor den umstebenden Zeugen seine Behauptung vor. Die wilden Stämme Indiens betrachten den Baumwollenstrauch als den Aufenthaltsort noch strengerer Gottheiten; da sie indess nur ihre nächste Umgebung bewachen, folglich minder beschäftigt sind, so haben sie auch mehr Musse, die Wahrhaftigkeit derer, die sich ihnen nahen, aufmerksam zu erforschen."

Der Papaya aber ist, dem Glauben der Hindu zufolge, die Residenz einer der drei das Weltall regierenden Glieder der indiscben Dreieinigkeit, während der Baumwollenstrauch und einige andere Büume die Olympe untergeordneter Götter sind, deren Mission lediglich darin besteht, das Leben und Treiben der Menschen in. nerhalb eines Districtes, oft auch nur eines einzigen Dorfes zu überwachen. (Ausland. 1859. S. 479.)

\section{Ueber die Vegetationsverhältnisse in Nizza.}

Nizza verdankt sein mildes Klima der südlichen Lage und dem Schutze einer dreifachen Bergterrasse der Seealpen gegen die kalten Nordwinde. Die Vegetation ist in Folge davon eine mehr südliche, und selbst tropische Pflanzen überdauern den Winter im Freien. Nur ausnahmsweise sinkt das Thermometer im Januar unter Null, steht im Winter überhaupt am 'Tage auf 12-17 Grad, dabei sind 220 Tage im Jahre ganz klar. Der Nordwestwind aus der Provence drückt die Temperatur etwas herunter, aber immer nur auf wenige Tage. Vom Meeresstrande aus erstreckt sich eine unbedeutende Ebene, in der nur Gartenbau betrieben wird und worin die Agrumen und sonstigen Obstbäume stehen, auch die im Freien ausdauernden exotischen Zierptlanzen. Diese Region reicht noch in dic nächste Bergreibe hinein, welche mit Reben, Feigen und Olivenbäumen besetzt ist, unter deren Schutze sämmtliche Cerealien des mittägigen Europas gedeihen. Höher folgen die Seefichten. Die nordischen Obst- und Waldbäume verlieren meist gegen Weihnachten ihre Blätter, aber blühende Pflanzen giebt es um diese Zeit viele. Rosen, Pelargonien, Daturen, spanische Kresse u. a. treiben unausgesetzt Blüthen. Ende Januar treiben Flieder und Thränenweide Blätter, Veilchen und Jonquillen erscheinen, und grüne Gemüse, wie Artischocken, Erbsen und Blumenkohl, die Blüthen des Mandelbaumes brechen auf. Im Februar entfalten eich Pfirsich-, Aprikosen-, Kirsch- und Pflaumenbäume, auch der Lorberbaum. In kalten Jahren verzögert sich die Vegetation allerdings um einige Wochen, indem die Nachtfröste im Januar die zarten Knospen zerstören. Viele tropiscbe Arten haben sich jedoch vollständig acclimatisirt. Unter diesen sind vorzugsweise folgende sehr charakteristisch: Acacia lophanta, Agave americana, die häufig bis 15 hohe Blüthenstengel treibt, Amaryllis formosissima, Amomum Zerumbet, Anona cherinolia, Caesalpina sappas, Calla aethiopica, Chamaerops humilis, Citrus aurantium in allen Gärten und 
neuerdings zu einem sehr wohlechmeckenden Weine verwerthet, Cereus grandiflorus, Diospyros japonica, Dracaena draco, Ebenus creticus, Erythrina crista galli, Eugenia janbosa, Ficus elastica, Glycine chinensis, Gossypium herbaceum, G. arboreum in den Gärten als Seltenheit, Heliotropum peruvianum, $H$. grandifiorum, Hibiscus Manihot, Hoya carnosa, Illicium anisatum, Justitia adhatoda, J.speciosa, Laurus benzoin, camphora, cinnamomum, indica, sassafras, Magnolia grandiflora, Melia azedarach, Melaleuca hypericifolia, Metrosideros lophanta, Mimosa pudica, Musa paradisiaca (nur bei grosser Pflege reife Früchte tragend), Nicotiana glauca (als hübscher schnellwüchsiger Baum), Opuntia vulgaris, $O$. cochenillifera (die Cochenillewürmer gingen zu Grunde), Phoenix dactylifera, ohne schmackhafte Friichte zu liefern, Phytolacca arborea, Ph. decandra, als schöner grosser Stamm, Plumbago ceylonica, Polygala arborea, nur mässig, aber dicht mit blauen Schmetterlingsblumen bedeckt vom October bis ins Frühjahr, Psidium pomiferum, $P . p y$ riferum, Rhus vernix, Saccharum officinarum nur spärlich, Schinus molle mit seiuem gefiederten Laube als schöner Alleebaum, Sola. num betaceum, Sparrmannia africana, Stapelia grandiflort, Styrax officinale, Thea viridis, Volkameria japonica, Yucca alaefolia, gloriosa. Im Garten des Grafen Cessole werden Ananas und Camellien in geöffneten Sommerhäusern gezogen. Aber ein Mangel ist in den Gürten, dass nicht mehr auf vorzüglich schöve Zierstrüucher und Bäume gehalten wird, durch die sich nur einzelne besonders auszeichnen. Die Nizzaer Flora steht überhaupt der von Neapel nicht nach, nur in Palermo tritt eine höhere Stufe ein, indem dort in freier Luft ausdauern: Musa paradisiaca und sapientum, Bambusa amondinacea, E'vythrina corollodendron, die die Stärke unserer Linden erreicht. (Berl.Gartenbaugesellsch. VI. 243-250.) Bkb.

\section{Zur Technologie.}

\section{Ueber Knapp's neue Methode zur Gerberei des Leders.}

Knapp hat durch eine grosse Reihe von Versuchen ein ganz neues Licht über den Process der Gerberei und die Constitution des Leders verbreitet. Die ältere Anschaungsweise wird dadurch gänzlich entkräftet, und nach $K n$ a p ist jede Art von Gerberei vielmehr ein rein mechanischer Process und das Leder ist das $R e$ sultat der blossen Zwischenlagerung des Gerbemittels zwischen die Fasern der Haut, welche dadurch an dem Zusammenkleben verhindert werden.

Knapp's Methode dieser neuen Art von Gerbung besteht in Folgendem.

Man bereitet zwei Bäder, eins mit Seifenwasser und eins mit gereinigter Salzlösung. $\mathrm{Zu}$ dem Seifenbade ist ihrer vollständigen Auflösljchkeit wegen Schmierseife besser, als die in der Kälte nur theilweise lösliche gewöhnliche harte Seife. Gemeine Schmierseife beeiuträchtigt jedoch die Reinheit der Farbe des Leders einigermaassen, was bei gewöhnlicher Sodaseife nicht der Fall ist. Wo es daher besonders darauf ankornmt, eine reine Farbe zu haben, wie beim weissen Alaunleder, thut man am besten, eine Schmierseife aus Kalilauge und reinem Fett (Talg etc.) zu verwenden. Die Seifenbäder müssen verdünnte sein, d. h. nicht mehr als $1 / 20 \mathrm{bis} 1 / 3$ 